Rep. of Tanzania; ${ }^{2}$ Centers for Disease Control and Prevention, Tanzania, United Rep. of Tanzania; ${ }^{3}$ Muhimbili University of Health and Allied Sciences MUHAS, Tanzania, United Rep. of Tanzania; ${ }^{4}$ Centres for Disease Control and Prevention, Atlanta, USA; ${ }^{5}$ Centres for Disease Control and Prevention, Tanzania, United Rep. of Tanzania

Background In Tanzania, adult HIV prevalence is estimated at 5.8\% and $9.3 \%$ in Dar es Salaam, well above the level of a generalised epidemic. Nonetheless, HIV infection and a constellation of associated factors are disproportionately borne by marginalised groups within the country. Female sex workers (FSW) are one such group; however, little is known about them in Tanzania.

Method Respondent-driven sampling was used to recruit self-identified FSWs aged $15+$ years, living in Dar es Salaam, who reported exchanging sexual intercourse for money in the past month. FSWs were interviewed about their social circumstances, STI risk behaviours and tested for HIV, Hepatitis B, C and other STIs. Point estimates and 95\% CI were adjusted for social network size and recruitment patterns using RDSAT.

Results From April to August 2010, 537 FSWs were recruited. Their median age was 29 years (range 15-63). Two FSWs (0.6\% 95\% CI: 0\% to $1.7 \%$ ) were $<10$ years when first selling sex (median age 17 years). Condom use at last sex was $82.1 \%$ (95\% CI: $77.0 \%$ to $87.0 \%$ ), but consistent use varied by type of partner; always condoms use with steady partner was $29.9 \%$ (95\% CI: $22.8 \%$ to $37.6 \%)$ and $65.1 \%(95 \%$ CI: $58.4 \%$ to $71.4 \%$ ) with one time client. The major reason for FSWs not using condoms was partner objection (44.1\%; $95 \%$ CI: $32.6 \%$ to $56.2 \%)$. The prevalence of STIs and viral hepatitis varied: HIV $30.2 \%$ (95\% CI: $24.7 \%$ to $36.4 \%$ ); Hepatitis B $5.6 \%$ (95\% CI: 3.7\% to $8.3 \%$ ); Hepatitis C $1.4 \%$ (95\% CI: $0.3 \%$ to $3.1 \%$ ), syphilis $2.1 \%$ (95\% CI: $0.8 \%$ to $3.4 \%$ ); Gonorrhoea $10 \%$ (95\% CI: $6.9 \%$ to $14.4 \%$ ), and Chlamydia $6.9 \%$ (95\% CI: $3.2 \%$ to $11.9 \%$ ). Many (292; $51.7 \%, 95 \%$ CI: $46 \%$ to $58 \%$ ) FSWs had experienced physical violence in the past 12 months and $34.7 \%$ (95\% CI: $28.7 \%$ to $40.6 \%$ ) had been forced to have sex. Few FSWs were IDU themselves (1.4\%; 95\% CI: $0.3 \%$ to $2.9 \%$ ), but $5.1 \%$ (95\% CI: $2.3 \%$ to $7.7 \%$ ) suspected regular clients, and $8.9 \%$ (95\% CI: $7 \%$ to $12 \%$ ) suspected one-time clients were IDU. These women had significantly higher prevalence of HIV (46.8\%; $95 \%$ CI: $31.5 \%$ to $63.9 \%$ compared to $23.1 \%$ (95\% CI: $16.4 \%$ to $29.3 \%$ ) for FSWs who did not suspect any partner.

Conclusion FSWs of Dar es Salaam have many-fold higher prevalence of HIV than the general population, with variable levels of structural interventions beyond HIV education and condom promotion. Preventive, care and treatment and personal level empowerment strategies are desperately needed among FSWs in Dar es Salaam, Tanzania.

\section{1-S08.02 WHO ARE THE WOMEN AT RISK OF HIV INFECTION IN RURAL ZIMBABWE AND HOW MANY ARE THERE? INSIGHTS INTO THEIR CHARACTERISTICS, LOCATIONS, AND BEHAVIOURS}

doi:10.1136/sextrans-2011-050109.44 other STIs. Their health situation calls for multifaceted services and

any form of sexual exchange motivated by material reward including cash-per-act and informal credit-based arrangements. We restricted our analysis to a subset of WR who report receiving money for sex, excluding non-monetary transactions. WR were assigned to high, medium or low activity levels based on, among other factors, the frequency they report receiving money for sex and numbers of partners. CIs are based on Agresti-Coull 95\% estimation and $p$ values are for $\chi^{2}$ estimation unless otherwise stated.

Results WR were more common in commercial centres than in rural areas $11.1 \%$ (9.9\% to $12.4 \%$ ) vs $5.0 \%$ (4.3\% to $5.8 \%$ ). The pattern of sex work activity differed between locations $(p=0.049)$ and mean number of sexual partners, over 2 weeks, in high, medium and low activity WR were 4.1, 1.9 and 1.3 respectively (ANOVA test for trend: $\mathrm{p}<0.001)$. High activity WR comprised $2.4 \%(1.9 \%$ to $3.1 \%)$ of women in commercial centres vs $0.55 \%(0.34 \%$ to $0.89 \%)$ in rural; medium level, $7.1 \%$ (6.2\% to $8.2 \%$ ) vs $3.9 \%$ (3.3\% to $4.7 \%$ ); and low level, $1.56 \%(1.13 \%$ to $2.13 \%)$ vs $0.50 \%(0.30 \%$ to $0.82 \%)$,Abstract O1-S08.02 figure 1. Overall, HIV prevalence in WR was higher in commercial centres than in rural areas: $46.5 \%$ (95\% CI: $38.9 \%$ to $54.3 \%$ ) vs $37.8 \%$ ( $28.4 \%$ to $48.1 \%$ ) ( $p>0.1$ ). Mean HIV prevalence among high level WR was consistent across socioeconomic strata: 50\% (range: $35.8 \%-64.2 \%$ ). Prevalence among medium level WR tended to be higher in business centres: $47.5 \%$ (38.1\% to $57.2 \%)$ vs $35.2 \%$ (25.1\% to $46.8 \%$ ) in rural areas. In contrast, HIV prevalence in low level WR was higher in rural areas: $44.4 \%$ (18.8\% to $73.4 \%)$ vs $36.4 \%$ (19.6\% to $57.1 \%)$.
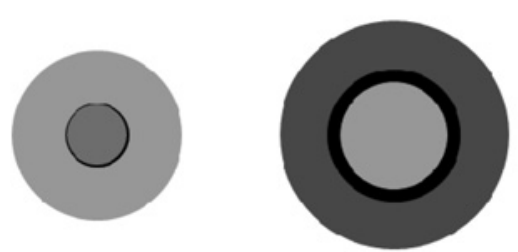

HIV prevalence (\%)

\section{Rural areas Commercial centres}

Abstract 01-S08.02 Figure 1 Relative size and HIV prevalence of the WR population in two socioeconomic locations in east Zimbabwe. Circle sizes are in proportion to the relative size of WR activity level; the inner ring represents low activity, the central ring high activity and the outer ring medium activity WR.

Conclusions Sex worker populations may be larger than previously measured in general population surveys in rural Zimbabwe. Given that HIV prevalence among WR is threefold higher than in the general population and the underestimated size of the sex worker population, sex work may be an increasingly important driver of HIV transmission in declining generalised rural African epidemics.

\section{1-S08.03 CONDOM USE DURING WORK TIME AMONG FEMALE SEX WORKERS IN BENIN}

doi:10.1136/sextrans-2011-050109.45

\begin{abstract}
${ }^{1} \mathrm{~J}$ Elmes, ${ }^{2} \mathrm{~K}$ Nhongo, ${ }^{1} \mathrm{~T}$ Hallett, ${ }^{1} \mathrm{P}$ White, ${ }^{2} \mathrm{R}$ Mutsindiri, ${ }^{1} \mathrm{G}$ Garnett, ${ }^{2} \mathrm{C}$ Nyamukapa, ${ }^{1} \mathrm{~S}$ Gregson. ${ }^{1}$ Imperial College London, London, UK; ${ }^{2}$ Biomedical Research and Training Institute, Zimbabwe
\end{abstract}

Background In Zimbabwe's generalised epidemic, the relative importance of different risk groups to ongoing HIV transmission is unclear. We estimated population sizes and HIV prevalence of women involved in different levels of sex work activity in two socioeconomic locations (rural areas and commercial centres) in east Zimbabwe

Methods The baseline survey for a cohort study of 650 women at high risk of HIV infection (WR) was conducted in March 2010, using snowball and location-based methods to recruit women with
${ }^{1} \mathrm{~S}$ Diabaté, ${ }^{1} \mathrm{~A}$ Chamberland, ${ }^{2} \mathrm{~N}$ Geraldo, ${ }^{3} \mathrm{D} \mathrm{M}$ Zannou, ${ }^{4} \mathrm{M} \mathrm{M}$ Loembé, ${ }^{5} \mathrm{~S}$ Anagonou, ${ }^{6} \mathrm{~A}$ C Labbé, ${ }^{7} \mathrm{C}$ Tremblay, ${ }^{8} \mathrm{M}$ Alary. ${ }^{1}$ Centre de recherche, Centre hospitalier de I'Université de Montréal, Montréal, Canada; ${ }^{2}$ Dispensaire IST, Centre de santé de Cotonou I, Cotonou, Benin; ${ }^{3}$ Centre national hospitalier universitaire de Cotonou, Cotonou, Benin; ${ }^{4}$ Institut de Médecine tropicale, Anvers, Belgium; ${ }^{5}$ Centre national hospitalier universitaire, Cotonou, Benin; ${ }^{6}$ Université de Montréal, Hôpital Maisonneuve-Rosemont, Montréal, Canada; ${ }^{7}$ Centre de recherche du Centre hospitalier de I'Université de Montréal, Montréal, Canada; ${ }^{8}$ URESP, Centre de recherche FRSO du CHA universitaire de Québec, Québec, Canada

Background Condom use remains the cornerstone of HIV prevention in sub-Saharan Africa where HIV transmission is 